\title{
Aceptabilidad a suplementos nutricios en mujer es embarazadas o lactando y niños menores de cinco años
}

\author{
Homero Martínez, M. C., Ph. D., ${ }^{(1)}$ Lourdes Campero, M. en Educ., ${ }^{(1)}$ \\ Guadalupe Rodríguez, M. en Admón., (1) Juan Rivera, Ph.D..11
}

\begin{abstract}
Martínez H, Campero L, Rodríguez G, Rivera J. Aceptabilidad a suplementos nutricios en mujeres embarazadas o lactando y niños menores de cinco años. Salud Publica Mex 1999;41:163-169.
\end{abstract}

\begin{abstract}
Resumen
Objetivo. Se llevó a cabo un estudio exploratorio para profundizar en aspectos relativos a la aceptabilidad y el apego al consumo de suplementos nutricios que forman parte de un programa gubernamental de apoyo a la nutrición, dirigido a grupos vulnerables. Material y métodos. Se entrevistó a mujeres embarazadas o en lactancia, así como a madres de niños lactantes, niños destetados y niños menores de dos años con desnutrición. Los datos se recolectaron mediante visitas al hogar, aplicando una guía de entrevista con formato libre. Las entrevistas se grabaron previo consentimiento informado de las mujeres. El análisis incluyó temas y subtemas que abordaron las entrevistadas. Resultados En general hubo una buena aceptabilidad al suplemento cuando se ofreció en forma de bebida. Al considerar sus beneficios, las mujeres embarazadas antepusieron la salud del bebé a la propia y asociaron el valor del suplemento con su contenido de "vitaminas". La aceptación a los sabores que se les ofrecieron se relacionó con la costumbre de consumirlos. Los niños menores de un año de edad prefirieron la consistencia líquida, y los mayores, la papilla. La opinión general fue que la cantidad ofrecida er a suficiente, y se comentó que conforme transcurría el tiempo de intervención, aumentaba el apetito. N o hubo efecto de sustitución de la dieta por el suplemento. Conclusiones La aceptabilidad al suplemento puede fortalecerse mediante mensajes orientados al bienestar y la salud del niño, introduciendo la noción de que el suplemento también es benéfico para la mujer gestante. Se recomienda involucrar a los proveedores de salud de las comunidades a fin de que ayuden a lograr un mejor consumo y aceptabilidad del producto. La identificación del contenido de "vitaminas" por parte de las
\end{abstract}

\author{
Martínez H, Campero L, Rodríguez G, Rivera J. \\ Acceptance of nutritional supplements among \\ pregnant and lactating women \\ and children under five years of age. \\ Salud Publica Mex 1999;41:163-169.
}

\begin{abstract}
A bstract
Objective.The present study explo res the acceptance and consumption of nutritional supplements that form part of a governmental program to support nutritionally vulnerable groups. Material and Methods Pregnant and lactating women, and mothers of malnourished toddlers, infants and children were interviewed. Data were collected, after introduction with an interview guide with an open-ended format, through face-to-face interviews conducted at home. Interviews were taped with previous informed consent. Analysis included topics and subtopics approached by the interviewed women. Results. The studied population showed good acceptance to the supplement when offered as a drink. When considering benefits, pregnant women thought first of their baby's health than of their own and associated the possible advantages of the supplement to its "vitamin" contents. Acceptance of the flavors was largely influenced by previous contact to specific flavors. Children under 1 year of age preferred the liquid consistency and elderly children favored the puree. Most mothers considered that the offered amount was enough and there was the general impression that, as the time of the intervention elapsed, children showed greater appetite.The diet was not substituted by the supplement. Conclusions.Acceptance of the supplement may be improved by messages focusing on the well-being and health of the child and insisting on the benefits for the pregnant mother. Community health providers should be involved in supporting the program, recommending consumption and acceptance of the supplement. The identification by mothers of "vitamins" as part of the supplement may be used to reinforce the concept of beneficial effects associated to micronutrient supplementation.
\end{abstract}

(1) División de Investigación en Salud y N utrición, Instituto N acional de Salud Pública, México.

Fecha de recibido: 15 de septiembre de 1998 - Fecha de aprobado: 2 de marzo de 1999

Solicitud de sobretiros: Dr. Homero Martínez. Instituto N acional de Salud Pública, División de Investigación en Salud y N utrición.Av. Universidad 655, cololonia Santa María A huacatitlán, 62508 Cuernavaca, Morelos, México.

Correo electrónico: homero@ solar.sar.net 
madres puede utilizarse para reforzar el concepto de los beneficios del consumo de micronutrientes. Es importante llevar a cabo evaluaciones cualitativas como parte de la evaluación de proceso del programa.

Palabras clave: alimentación suplementaria; embarazo; lactancia materna; lactante; infante; México
Q ualitative evaluations should be performed as part of the assessment of community-based programs.

Key words: supplementary feeding; pregnancy; breast feeding; infant; child, preschool; Mexico
E n los países en desarrollo, la mayor prevalencia de desnutrición se presenta en las familias del medio rural. ${ }^{1}$ Por otro lado, al interior de la familia existen grupos en los que la desnutrición se presenta con mayor frecuencia y en quienes las consecuencias son más graves; esos grupos vulnerables están representados por las mujeres embarazadas y en lactancia, así como por los niños de 4 a 24 meses de edad.

Existen diversas estrategias para atender la desnutrición en esos grupos, entre las que se encuentran la educación nutricia, la fortificación de alimentos y la suplementación nutricia., ${ }^{2,3}$ Entre las ventajas de esta última están la de poder focalizar la intervención hacia los grupos en riesgo y la inclusión de varios nutrientes como parte de la misma. Un aspecto fundamental para el éxito de la intervención, así como para garantizar su sostenibilidad, es que la población blanco tenga una buena aceptabilidad al suplemento.

El objetivo de este trabajo fue explorar y profundizar en aspectos relacionados con la aceptabilidad al consumo de suplementos nutricios por parte de mujeres embarazadas y en periodo de lactancia, así como por niños de 4 a 24 meses de edad, residentes en una comunidad rural del estado de Morelos.

\section{Material y métodos}

El estudio se llevó a cabo en la comunidad de Xoxocotla, Morelos, durante el segundo semestre de 1997. A partir de una muestra estratificada en la que se exploraron la aceptación y el apego al consumo de dos formulaciones de suplemento nutricio, ${ }^{4}$ se seleccionó, en forma intencionada para este estudio, una muestra de mujeres embarazadas y en lactancia, así como de madres de niños de 4 a 24 meses de edad, y de madres de niños mayores de 24 meses que presentaran algún grado de desnutrición, definida por un peso para la talla menor a una desviación estándar (-1 DE) del patrón de referencia del National Center for Health Statistics. ${ }^{5}$ Se trata de los grupos de población en riesgo característicos, a quienes va dirigido el suplemento. Para este estudio exploratorio a profundidad se optó por un tamaño de muestra de siete mujeres embarazadas, seis mujeres en periodo de lactancia y sus hijos, siete madres de niños ya destetados y menores de dos años de edad y cinco madres de niños ya destetados, mayores de dos años de edad y con algún grado de desnutrición, para un total de 31 entrevistas. El tamaño de la muestra quedó determinado por su factibilidad en la conducción del estudio. La muestra correspondió a poco más de $10 \%$ del total de sujetos participantes en el estudio de base, orientado a la medición de la aceptación y del apego al consumo del suplemento nutricio (estudio de efectividad).

Para la selección de los participantes se identificaron características que representaran una diversidad en la aceptabilidad al suplemento, con el objeto de identificar aspectos extremos de la aceptación y el apego al producto ofrecido. Con base en dicho criterio, la muestra de mujeres embarazadas y en lactancia se formó con: aquellas que habían asistido regularmente al centro de suplementación de la comunidad y con quienes asistieron de manera irregular, o bien, que se hubieran dado de baja; mujeres que no presentaran problemas de salud durante el estudio y mujeres que expresaron tener algún problema que relacionaban con la toma del suplemento; mujeres que expresaron algún comentario, positivo o negativo, sobre el producto al recibirlo y, por último, mujeres a quienes se dieron bebidas con sabor de vainilla, de plátano o sin sabor.

La muestra de madres de niños participantes se seleccionó aplicando los mismos criterios anteriores; se confirmó que, además de haber recibido los diferentes sabores del suplemento, también se les hubiese proporcionado en forma de papilla o de bebida.

En síntesis, se trató de seleccionar una muestra que representara la variedad de tipos de mujeres embarazadas y madres que participaron en este estudio. Como se ha señalado reiteradamente en la literatura especializada, ${ }^{6,7}$ el valor de la investigación cualitativa no radica en la representatividad estadística de sus hallazgos, sino en la posibilidad de profundizar en la comprensión de los procesos sociales estudiados. La investigación cualitativa permite caracterizar las va- 
riaciones de las conductas de los individuos, e identificar en sus motivos, valoraciones y apreciaciones subjetivas, la lógica de esas variaciones. ${ }^{8}$

La recolección de datos se llevó a cabo mediante visitas domiciliarias, todas a cargo de una psicóloga con experiencia previa en la aplicación de este tipo de entrevistas en campo. En esta visita se aplicó una entrevista, basada en una guía de temas, de tal forma que la entrevistada pudiera hablar libremente, sin un formato estructurado. ${ }^{9-11}$ La guía incluyó información sobre la identificación de la entrevistada, su dieta habitual, las expectativas que tenía sobre el suplemento nutricio, la aceptación a la consistencia y el sabor del producto, y el apego al consumo del mismo. En el caso de la entrevista con las madres de niños, se incluyó información sobre edad, prácticas de ablactación y aparición del brote dentario.

Con el previo consentimiento informado de las mujeres se grabaron todas las entrevistas y después se transcribieron textualmente. Para analizar la información se elaboró un índice de los temas y subtemas que abordaron en forma directa los sujetos entrevistados, así como los que se generaron de manera indirecta. ${ }^{6}$ Con base en la codificación establecida se elaboró un instrumento para favorecer la identificación de las relaciones y diferencias entre las diversas ideas, según los informantes. Por último, de acuerdo con esta información, se sistematizó con mayor detalle el agrado o desagrado que se manifestó hacia el suplemento, es decir, las particularidades que conformaron su aceptación y apego. ${ }^{12}$

\section{Resultados}

\section{Descripción de la población}

Mujeres embarazadas. La mayoría estaba en su segundo embarazo, cursando su segundo trimestre de gestación, con pareja conyugal formal; formaban parte de familias extensas y eran amas de casa.

Mujeres lactando. La mayoría eran madres primigestas, al final del primer año de lactancia, con pareja conyugal; vivían en familias extensas y eran amas de casa. Niños lactantes. Se incluyeron niños de entre 4 y 18 meses de vida, todos parte de familias nucleares. Sus madres eran amas de casa. Aún no contaban con dentición completa, pues les faltaban algunos colmillos o molares, pero ya habían iniciado la ablactación, con frutas, sopas, caldos y atoles de avena o arroz. Al momento de la encuesta los niños recibían alimentos "delgaditos, licuados o moliditos".
Niños destetados, menores de dos años. Las edades de este grupo se encontraron entre los 12 y los 24 meses. Los niños vivían en familias extensas. Algunas madres estaban separadas de su pareja, por lo que debían trabajar para sostener a su familia. Los niños contaban con casi toda su dentición, con excepción de algunos molares. El inicio de su ablactación fue a base de frutas, sopas o caldos y atoles. Las madres refirieron haber lactado al seno materno al menos durante su primer año de vida. Al momento de la encuesta los niños comían "casi de todo".

Niños destetados mayores de dos años, con desnutrición. Las edades de este grupo se encontraban entre los 2 y 3 años de vida. Los niños vivían en familias extensas, y sus madres eran amas de casa. Contaban ya con dentición completa, habían recibido lactancia materna al menos durante su primer año de vida, y el inicio de su ablactación fue similar al descrito para los grupos anteriores. Al momento de la encuesta las madres señalaron que los niños "comían de todo".

\section{Opiniones de las mujeres embarazadas y en lactancia}

Sobre la consistencia, el sabor y la cantidad del suplemento. Las opiniones de las mujeres embarazadas y en periodo de lactancia sobre la consistencia, el sabor y la cantidad de la bebida fortificada se presentan en el cuadro I. En el caso de las mujeres embarazadas, cabe destacar que en un inicio les costó trabajo acostumbrarse a la consistencia de la bebida, pero en la medida en que pasó el tiempo la aceptaron. La principal razón que señalaron fue que no tomaban la bebida por gusto, sino porque reconocían que "era bueno para el bebé." Aunque originalmente se consideró ofrecer sólo el sabor natural, en la fase inicial de la prueba de campo todos los sabores (plátano, vainilla y natural) fueron bien aceptados, y en general hubo más comentarios positivos que negativos hacia las bebidas. Además, algunas mujeres que tomaron el sabor natural comentaron que lo sentían "simple", en contraste con las bebidas con sabor. Varias mujeres asociaron la bebida con la leche, y comentaron que estaban acostumbradas a agregarle algún sabor. Respecto a la cantidad ofrecida diariamente en el centro de suplementación, las mujeres opinaron que estuvo bien y que aceptarían seguir tomándola, ya que una vez más reconocieron los beneficios para el bebé. Desde un inicio las mujeres lactando mostraron una mayor aceptación y agrado en relación con la consistencia de la bebida. Este grupo aceptó igualmente el sabor a plátano y vaini- 
lla; en cuanto al natural, algunas mujeres expresaron que estaban de acuerdo con que el sabor fuera "simple", debido a que estaban dando pecho y acostumbraban tomar la leche sin sabor. El grupo entrevistado opinó que la cantidad de leche ofrecida era poca.

Sobre el contenido nutricio y los beneficios o problemas. La opinión de las mujeres embarazadas y lactando sobre el contenido nutricio y los beneficios o problemas esperados por el consumo de la bebida fortificada, se presenta en el cuadro II. Tanto unas como otras identificaron a las vitaminas como el elemento central de la bebida y como un componente positivo sobre todo para el desarrollo de su bebé. Algunas mujeres embarazadas identificaron riesgos de consumo de vitaminas en el embarazo, pensando que podía ocasionar un crecimiento mayor o desmedido del bebé, que complicaría el parto. Por otro lado, algunas mujeres embarazadas tenían el antecedente de haber tomado vitaminas en otros embarazos, aunque no de manera regular, en tanto que las multigestas lactando no habían tenido la experiencia de tomar vitaminas durante la lactancia de sus otros hijos. Estas mujeres no identificaron ningún riesgo en el consumo de vitaminas durante el periodo de lactancia.

Opinion de las mujeres sobre el apego. La opinión de las mujeres embarazadas y lactando sobre el apego al consumo de la bebida fortificada se presenta en el cuadro III. Varias mujeres comentaron que al inicio de la intervención les dio más hambre; por ejemplo, decían "se me abrió el apetito". Hubo también algunas embarazadas que refirieron que cuando comenzaron a tomar la bebida, ésta les caía muy pesada, y hubo quien comentó que le ocasionó diarrea. Las mujeres embarazadas tuvieron una asistencia más irregular, y aunque contaban con el apoyo por parte de su pareja, hubo también algunas que tuvieron problemas con sus esposos por asistir al centro de suplementación, pues ellos consideraban que descuidaban su casa. Las mujeres lactando no refirieron tener problemas en casa por asistir al centro. Algunas embarazadas indicaron que diversos proveedores de salud, principalmente parteras, les habían comentado que al tomar un suplemento corrían el riesgo de tener un bebé muy grande, con posibles complicaciones en el parto. Las mujeres lactando también comentaron que algunos proveedores de salud o familiares les habían dicho que el bebé crecería más, pero en este caso esto no fue visto como desventaja.

\section{Opiniones de las madres}

Opinion sobre la aceptabilidad de sus hijos. En el cuadro IV se presentan las opiniones de las madres sobre la consistencia, el sabor y la cantidad ofrecida de bebida o papilla a los niños. Los lactantes prefirieron la presentación líquida del suplemento, en tanto que a los dos grupos de niños destetados les gustó más la papilla. El sabor a plátano fue el que tuvo más aceptación en todos los grupos, seguido por el de vainilla. El sabor a chocolate fue aceptado en el grupo de niños mayores de dos años, quienes acostumbraban agregar una bebida comercial (Choco Milk) sabor chocolate a su leche. En relación con la cantidad, únicamente las madres de niños menores de dos años, ya destetados opinaron que era poca.

Opiniones de las madres y familiares sobre el complemento y la salud de los niños. En el cuadro V se resume la opinión de las madres sobre la influencia de la bebida o

\section{Cuadro I \\ Opinión de las mujeres embarazadas y en lactanCia sobre La ACEPTACIÓn de LA bebida fortificada. Xoxocotla, Morelos, MéXico, 1997}

Grupo

Mujeres embarazadas $(n=7)$

Mujeres en lactancia $(n=6)$

\section{Consistencia}

La mayoría comentó en un inicio sobre la dificultad para tomar la bebida; p.e., "Está espesa y con harta grasa.".

La mayoría expresó, desde un inicio, el agrado que le ocasionaba tomar la bebida, p.e.: "El estómago me lo pide.".

\section{Sabor}

Plátano: recibió más comentarios positivos que negativos.

Vainilla: recibió más comentarios negativos que positivos.

$\mathrm{N}$ atural: recibió igual proporción de comentarios positivos que negativos.

Plátano: recibió más comentarios positivos que negativos.

Vainilla: recibió más comentarios negativos que positivos.
Cantidad ofrecida

La mayoría comentó que la cantidad de bebida ofrecida fue adecuada. Señalaron que, cuando tomaban leche, acostumbraban consumir un vaso, p.e.:"Yo tomo un vaso de leche diario, así que lo que me daban estaba bien.".

La mayoría comentó que la cantidad ofrecida fue poca. También comentaron que acostumbraban tomar más de un vaso de leche diario, p.e.: "Yo me tomaría el doble de lo que me dan.". 


\section{Cuadro II \\ Opinión de la mujeres embarazadas y en lactancia sobre el CONTENIDO NUTRICIO de la bebida fORTIFiCADA y sus efectos en la Salud. Xoxocotla, Morelos, México, 1997}

\begin{tabular}{lll}
\multicolumn{1}{c}{ Grupo } & $\begin{array}{c}\text { Identificación del contenido } \\
\text { principal del producto }\end{array}$ & Identificación de beneficios \\
$\begin{array}{l}\text { Mujeres embarazadas } \\
(n=7)\end{array}$ & Vitaminas & $\begin{array}{l}\text { La mayoría indicó que el su- } \\
\text { plemento servía para que "se } \\
\text { desarrolle bien el bebé". }\end{array}$ \\
$\begin{array}{l}\text { Mujeres en lactancia } \\
(n=6)\end{array}$ & Vitaminas & $\begin{array}{l}\text { La mayoría comentó que el } \\
\text { suplemento servía para "ali- } \\
\text { mentar mejor al niño". }\end{array}$
\end{tabular}

Identificación de riesgos por
tomar el suplemento

La mayoría expresó percibir un riesgo de que el bebé creciera mucho y el parto se complicara, 0 sea que desembocara en cesárea.

$\mathrm{N}$ inguna identificó un riesgo específico asociado con el consumo de la bebida fortificada.

\author{
Antecedentes de consumo de \\ vitaminas en experiencias \\ similares
}

Sí había antecedentes, aunque con consumo irregular.

No había antecedentes de consumo de vitaminas durante la lactancia.

\section{Cuadro III \\ Opinión de las mujeres embarazadas y en lactancia sobre el apego al consumo de la bebida fortificada. Xoxocotla, Morelos, México, 1997}

\begin{tabular}{|c|c|c|}
\hline Grupo & $\begin{array}{l}\text { Percepción de cambios } \\
\text { en su alimentación }\end{array}$ & $\begin{array}{c}\text { Presencia de problemas } \\
\text { de salud }\end{array}$ \\
\hline $\begin{array}{l}\text { Mujeres embarazadas } \\
(n=7)\end{array}$ & $\begin{array}{l}\text { A lgunas mujeres co- } \\
\text { mentaron que al iniciar } \\
\text { el consumo de la bebi- } \\
\text { da les dio más hambre. }\end{array}$ & $\begin{array}{l}\text { Algunas mujeres co- } \\
\text { mentaron que, en el } \\
\text { inicio, la bebida les caía } \\
\text { mal, pesada, y tuvieron } \\
\text { diarrea. }\end{array}$ \\
\hline
\end{tabular}

Mujeres en lactancia La mayoría comentó $(n=6)$ zaron a tomar la bebida, tenían más hambre.

La mayoría de las mujeres no comentó ningún problema particular.
Asistencia y bajas

Mayor número de inasistencias 0 asistencia irregular. Refirieron tener problemas con el marido para poder asistir.

Mayor número de asistencia regular, y muy pocas bajas en este grupo.

\section{Opinión recibida de los familiares y proveedo- res de salud sobre el suplemento \\ Razones por las cuales otras mujeres dejaron de participar en el estudio}

Generalmente positivos, con reconocimiento de las ventajas para el desarrollo del bebé en formación.Algunas parteras opinaron que el bebé podía crecer demasiado, y complicar el parto.

Generalmente positivos, con reconocimiento de que el suplemento era útil para el crecimiento del niño.
Muy pocas refirieron saber por qué otras mujeres dejaron de participar. Entre las razones que sí mencionaron, predominó el disgusto por la consistencia o sabor de la bebida.

Muy pocas refirieron saber por qué otras mujeres dejaron de participar, aunque se mencionaron el disgusto por la consistencia o sabor de la bebida, y problemas de salud con sus hijos. papilla en la dieta habitual de los niños, la presencia de problemas de salud, y los aspectos relacionados con la asistencia al programa así como las opiniones de los familiares respecto al mismo. Un comentario generalizado fue que el suplemento les "abría el apetito" a los niños. Tanto en el grupo de lactantes como en el de mayores de dos años con desnutrición, el suplemento se tomó además de la dieta habitual, sin efecto de sustitución o desplazamiento. En el grupo de menores de dos años destetados, el consumo del suplemento fue irregular, pues en algunas ocasiones se realizó junto con la dieta y en otras desplazó a la alimentación habitual. Aunque bastantes niños presentaron problemas respiratorios o de diarrea a lo largo del estudio, la mayoría de las madres no los asociaron con el consumo del suplemento. En ningún caso se notificó algún problema de salud que ocasionara la baja del programa, y en general la asistencia fue muy buena. La opinión de los familiares respecto a la participación de los niños en el programa fue positiva y estimulante.

\section{Discusión}

Las mujeres embarazadas consideraron la toma del suplemento como "buena para el bebé", anteponiendo este principio al de su propia salud. También se 


\section{Cuadro IV \\ Opinión de las madres sobre la aCePTACión de la bebida fortificada entre sus hiJOS. XoXocotla, Morelos, México, 1997}

\begin{abstract}
Grupo
Consistencia

Sabor

Cantidad ofrecida

$\mathrm{N}$ iños lactantes de $6-18$ meses $(n=6)$

$\mathrm{N}$ iños destetados menores de 2 años $(n=7)$

$\mathrm{N}$ iños destetados de 2 a 3 años de edad y desnutridos $(n=5)$

La mayoría prefirió la bebida a la papilla.

La mayoría prefirió la papilla a la bebida.

La mayoría prefirió la papilla a la bebida.

La mayoría prefirió el plátano o la
había probado antes el chocolate.

La mayoría prefirió el sabor a plátano o el de vainilla. Sólo una minoría prefirió el sabor a chocolate, a pesar de que todos ya lo habían probado con anterioridad.

La preferencia fue por los sabores a plátano y chocolate. Una mayoría acostumbraba consumir Choco Milk como parte de su dieta habitual. ofrecida era la adecuada. ofrecida era poca. ofrecida era la adecuada.

\section{Cuadro V \\ Opinión de las madres sobre el apego al consumo de la bebida o papilla fortificada por PaRte de sus hijos de entre 6 meses y 3 años de vida. Xoxocotla, Morelos, México, 1997}

Las madres opinaron que la cantidad

Las madres opinaron que la cantidad

Las madres refirieron que la cantidad
Grupo estudiado

$\mathrm{N}$ iños lactantes de 6-18 me$\operatorname{ses}(n=6)$

$\mathrm{N}$ iños destetados menores de 2 años $(n=7)$

$\mathrm{N}$ iños destetados mayores de 2 años, desnutridos $(n=5)$
Percepción de cambios en su alimentación

Comía lo de siempre, más el suplemento. Varias madres comentaron que conforme transcurrió la intervención, a sus hijos se les "abría el apetito".

Las madres comentaron que percibieron cambios en Su dieta, o sea, algunos días tomaban el suplemento y luego ya no querían comer y viceversa.

Las madres comentaron que comen "Io de siempre" más el suplemento. Varias madres comentaron que conforme transcurrió la intervención, a sus hijos se les "abría el apetito".
Presencia de problemas de salud

Asistencia y bajas

La mayo ría de los niños presentó problemas respiratorios durante el estudio. Algunas madres reportaron problemas de diarrea asociadas a la toma del suplemento, que no fue causa de abandono.

La mayoría de los niños presentó problemas respiratorios durante la intervención. Varios presentaron problemas "del estómago", aunque las madres no lo relacionaron directamente con la toma del suplemento.

La mayoría de los niños presentó problemas respiratorios durante la intervención. $\mathrm{N}$ inguna madre reportó problemas de salud asociados con la toma del suplemento.
La mayoría asistió regularmente. $\mathrm{N}$ o hubo bajas en el grupo estudiado.

La mayoría asistió regularmente. Hubo pocas bajas en el grupo estudiado.
Opinión recibida de los familiares y proveedores de salud sobre el suplemento

0 pinión positiva y estimulante para continuar con la asistencia.
En este grupo no se pudo evaluar la asistencia, puesto que se les visitaba a domicilio para dar el suplemento. Sin embargo, no hubo rechazo a la intervención.
0 pinión positiva y estimulante para continuar con la asistencia. encontró que identificaban la presencia de "vitaminas" como un elemento que ayudaba al crecimiento del bebé, aunque algunas madres se mostraron preocupadas por la posibilidad de que este crecimiento fuera excesivo y se tradujera en problemas durante el parto.

La aceptabilidad a los sabores parece estar influida por la costumbre. Así por ejemplo, las madres que acostumbraban tomar la leche sola preferían el sabor natural, y los niños cuyas madres acostumbraban agre- gar polvo con sabor a chocolate a su leche lo elegían en su bebida. También se encontró una clara preferencia por el sabor de plátano en todos los grupos, lo cual podría explicarse por la disponibilidad de esta fruta en la región.

Aunque al inicio las mujeres embarazadas se quejaron de la consistencia espesa de la bebida, una vez que se acostumbraron a la misma no hubo más problemas. Las madres consideraron más adecuada la bebi- 
da para los lactantes, y prefirieron la papilla para los niños destetados, lo cual podría relacionarse con la presencia de dentición completa en los niños mayores de un año.

La mayoría de las mujeres comentó que la cantidad que les ofrecían era adecuada, aunque algunas madres opinaron que era insuficiente. El hecho de que algunas mujeres que amamantaban consideraran insuficiente la cantidad, podría deberse a que tenían por costumbre consumir más de un vaso de leche diario. Las madres de niños destetados menores de dos años también comentaron que era poca la cantidad de suplemento que se ofreció a sus hijos. Esto podría explicarse por el hecho de que no se les llenaba el vaso que se usaba para ofrecer la bebida, lo que podría dar la impresión de que la cantidad era reducida. Los niños mayores de dos años y desnutridos no pidieron más papilla, quizá por la falta de costumbre de consumir mayores cantidades de alimento.

En la mayoría de los grupos hubo comentarios en el sentido de que, en la medida en que transcurría el tiempo de intervención, se incrementaba el apetito. Aunque en general no se notificó sustitución de la dieta por el suplemento, las mamás de niños menores de dos años ya destetados comentaron que en ocasiones sus hijos no querían comer en casa después de tomar la bebida, lo cual muy posiblemente se debió a que en ocasiones los niños acudían al centro antes de comer.

De acuerdo con estos resultados, se considera importante proponer que la aceptabilidad al suplemento en las mujeres embarazadas se fortalezca mediante mensajes orientados al bienestar y a la salud del niño. Sin embargo, sería conveniente introducir la noción de que el suplemento también es beneficioso para la mujer gestante. Debido a la importancia de sus opiniones sobre las prácticas de la población, es recomendable involucrar a los proveedores de salud de las comunidades, tales como enfermeras, parteras y médicos, dándoles información clara sobre la intervención, ya que su apoyo puede ser de gran utilidad para lograr un mejor consumo y una mayor aceptabilidad del producto $y$, a la vez, evitar incongruencias, dobles mensajes o contradicciones en lo recomendado.
El hecho de que la población identifique un contenido de "vitaminas" en el suplemento, así como sus efectos benéficos sobre el crecimiento, podría aprovecharse para reforzar el concepto de los beneficios que el consumo de micronutrientes genera. La variedad en sabores del suplemento puede ser buena, pues en general fueron aceptados, además de que evita el hastío en los consumidores.

Por último, se sugiere realizar este tipo de evaluación cualitativa sobre la aceptabilidad al producto como parte de la evaluación del proceso del programa, a fin de contar con retroalimentación cuando existen problemas de baja aceptabilidad a la intervención por grupos específicos de la población o por región.

\section{Referencias}

1. De 0 nís M, Monteiro C, A kre JG.The worldwide magnitude of proteinenergy malnutrition: A n overview from theW $\mathrm{HO}$ global database on child growth. Bull W orld Health O rgan 1993;71:703-712.

2. Maberly G, Trowbridge F, Yip R, Sullivan K, W est C. Programs against micronutrient malnutriton: Ending hidden hunger.Annu Rev Public Health 1994;15:277-301.

3.W orld B. Enriching lives: 0 vercoming vitamin and mineral malnutrition in developing countries.W ashington, D.C.:W orld Bank, 1994.

4. Rosado JL, Rivera J, López G, Solano L, Rodríguez G, Casanueva E et al. Desarrollo y evaluación de suplementos alimenticios para el programa de educación, salud y alimentación. Salud Publica Mex 1999;41:153-162.

5. Hamill P, Drizd T, Johnson C, Reed R. Monthly vital statistics report. Health examination survey data from the $\mathrm{N}$ ational $\mathrm{C}$ enter for $\mathrm{H}$ ealth Statistics (N CHS) 1976;25:1120.

6. G laser B, Strauss A. The discovery of grounded theory. Strategies for qualitative research. N ueva York:A Idine de Guyter, 1967.

7. Maxwell JA. Understanding and validity in qualitative research. Harvard Educ Rev 1992;62:279-300.

8. Pope $C$, Mays $N$. Reaching the parts other methods cannot reach:An introduction to qualitative methods in health and health services research. Br Med J 1995;311:42-45.

9. Reyna M, Díaz C. Saber hablar, poder hablar. El lenguaje en la vida cotidiana. México, D.F.: Universidad Autónoma Metropolitana, Unidad Xochimilco (Colección Modular), 1989.

10. D elgado J, Gutiérrez J. Métodos y técnicas cualitativas de investigación en ciencias sociales. Madrid: De Síntesis, 1995.

11. Spradley J.The etnographic interview. N ueva York: Holt, 1979.

12. Miles M. Q ualitative data analysis: An expanded source book. 2a. edición. Londres: Sage publications, 1994. 